University of Nebraska - Lincoln

DigitalCommons@University of Nebraska - Lincoln

$1-2000$

\title{
Quantum-Well Resistivity for Perpendicular Transport in Magnetic Layered Systems
}

\author{
Evgeny Y. Tsymbal \\ University of Nebraska at Lincoln, tsymbal@unl.edu \\ David G. Pettifor \\ University of Oxford, david.pettifor@materials.ox.ac.uk
}

Follow this and additional works at: https://digitalcommons.unl.edu/physicstsymbal

Part of the Condensed Matter Physics Commons

Tsymbal, Evgeny Y. and Pettifor, David G., "Quantum-Well Resistivity for Perpendicular Transport in Magnetic Layered Systems" (2000). Evgeny Tsymbal Publications. 18.

https://digitalcommons.unl.edu/physicstsymbal/18

This Article is brought to you for free and open access by the Research Papers in Physics and Astronomy at DigitalCommons@University of Nebraska - Lincoln. It has been accepted for inclusion in Evgeny Tsymbal Publications by an authorized administrator of DigitalCommons@University of Nebraska - Lincoln. 


\title{
Quantum-well resistivity for perpendicular transport in magnetic layered systems
}

\author{
E. Yu. Tsymbal and D. G. Pettifor \\ Department of Materials, University of Oxford, Parks Road, Oxford OX1 3PH, United Kingdom
}

(Received 4 August 1999)

\begin{abstract}
We show that quantum-well states enhance the current-perpendicular-to-planes resistivity of a metal film compared to the resistivity in the bulk at film thicknesses comparable with the mean free path due to the reduced number of conducting channels within the potential-well structure. This makes the mean free path an important parameter, which must be taken into account for the accurate treatment of results on currentperpendicular-to-plane giant magnetoresistance, rather than ignored by applying the two-current series-resistor model.
\end{abstract}

The phenomenon of giant magnetoresistance (GMR) has been observed in magnetic multilayers in two principal geometries: current in the plane (CIP) of the layers ${ }^{1}$ and current perpendicular to the planes (CPP). ${ }^{2}$ Although, due to the small multilayer film thickness, experiments within the CPP geometry are much more difficult, they can provide important information about the mechanisms of spin-dependent scattering.

Most experiments on CPP GMR are interpreted in terms of the two-current series-resistor (2CSR) model (e.g., Ref. 3 ), in which there are no relevant lengths except the layer thicknesses. According to this model the conductance of the multilayer can be calculated as a sum of conductances for the up-spin and down-spin electrons, each layer and each interface being considered as an independent resistor. As shown in Refs. 4 and 5, the 2CSR model is justified when the spindiffusion length is large compared to the layer thicknesses. The 2CSR model can be qualitatively understood by the argument that in the limit when the elastic mean free path $l_{\mathrm{mfp}}$ is short compared to the layer thicknesses each layer represents a separate resistor, and in the limit of a very long $l_{\mathrm{mfp}}$ the conductance is "self-averaging,", i.e., one should sum up scattering probabilities and this implies, like in the first case, resistors in series.

These models ${ }^{3-5}$ do not take into account any potential step at the interfaces arising from the difference in the potentials of the adjacent metallic layers. This adds new features to the electronic transport both within the CIP geometry $^{6}$ and the CPP geometry. ${ }^{7-9}$ In the case of CPP transport, it results in the interface resistance, ${ }^{7}$ which is a consequence of the specular scattering at the interface. Diffuse scattering by the interface disorder may either enhance or reduce the interface resistance, the latter occurring for large reflection coefficients. ${ }^{8}$ The interface resistance can be included in the 2CSR model. For noncollinear magnetic configurations spin-dependent potential steps cause the CPP GMR to deviate from a simple cosine function of angle between the magnetizations due to the interference between the coherent electron waves associated with the up-spin and down-spin electrons. ${ }^{9}$ However, this model reduces to the 2CSR model for collinear magnetic configurations, which is a consequence of the approximation of small potential steps assumed in this paper. In the cases where the 2CSR model fails to explain experimental data, a finite spin-diffusion length has to be introduced. ${ }^{10-12}$

If a metal film is placed between metals, which have higher electronic potentials, then quantum-well states are created within this film. As shown in Ref. 13, the quantumwell states in a nonmagnetic metal spacer layer, sandwiched between ferromagnetic layers, explain the oscillations in the interlayer exchange coupling. The quantum-well states are also responsible for the oscillations in CPP conductance and CPP GMR within the ballistic regime of conduction. ${ }^{14}$

In this paper we show that within the diffusive regime of conduction the quantum-well states result in an enhanced CPP resistivity of the metal film compared to its bulk resistivity, if the film thickness is comparable with the mean free path. The CPP resistivity of the film is defined as the relative average change in the film resistance $R$ with respect to its thickness $L$, i.e.,

$$
\rho(L)=\frac{\delta R(L)}{\delta L}
$$

We demonstrate that this effect has important consequences in spin-polarized transport, because it involves the mean free path, which is normally ignored when interpreting CPP GMR results.

Qualitatively the quantum-well resistivity can be understood by means of the free-electron model. Within this model the conductivity $\sigma$ is proportional to the number of conducting channels, i.e., the number of Bloch states moving in the direction of the current at the Fermi energy $E_{F}$ with different transverse momenta $\mathbf{k}_{\|}$perpendicular to the current. $^{15}$ This follows from the Drude formula, which can be represented as

$$
\sigma=\frac{2 e^{2}}{h} \frac{4}{3} N_{c} l_{\mathrm{mfp}},
$$

where $N_{c}$ is the number of the conducting channels per unit area and spin. In the bulk all the electrons with the transverse momenta $k_{\|} \leqslant k_{1}$ contribute to the current and $N_{c}$ is determined by the Fermi momentum $k_{1}=\sqrt{2 m E_{F}} / \hbar$, namely, $N_{c}=k_{1}^{2} / 4 \pi$. Attaching a layer of metal 2 with higher potential $U$ to both sides of a layer of metal 1, leads to the formation of quantum-well bound states in layer 1 . The bound 
states appear for those transverse momenta $\mathbf{k}_{\|}$, for which there are no states available in the adjacent layers of metal 2, i.e., for $k_{2}<k_{\|} \leqslant k_{1}$, where $k_{2}=\sqrt{2 m\left(E_{F}-U\right)} / \hbar$ is the Fermi momentum in metal 2 . The conducting channels, associated with these values of $\mathbf{k}_{\|}$, are closed in metal 1 , due to the specular reflection of electrons from the barriers at the interfaces. According to the Drude formula, the reduced number of conducting channels in the potential-well structure implies an enhanced resistivity in the metal layer compared to the bulk.

The resistivity of metal 1 in the potential-well structure is enhanced only when the thickness of the layer is less or comparable to the mean free path $l_{\mathrm{mfp}}$. The presence of disorder leads to the scattering and redistribution of the electrons between various $\mathbf{k}_{\|}$. This opens new conducting channels at distances greater than $l_{\mathrm{mfp}}$, which is the characteristic length for the change in $\mathbf{k}_{\|}$to occur. Opening the new conducting channels is connected with the involvement of the quantum-well states to the conduction. The quantum-well states, being localized within the potential well in the perfect structure, become delocalized at distances of the order of the $l_{\text {mfp }}$, due to the scattering by disorder, and therefore start to contribute to the conduction. Opening the new conducting channels reduces the resistivity of the metal layer with increasing thickness. At large layer thicknesses the currentcarrying electrons are distributed between various transverse momenta in accordance with the bulk band structure of this metal, the quantum-well states being completely delocalized, and the layer resistivity becomes equal to the bulk resistivity.

In order to treat this effect quantitatively we have performed calculations of the conductance $\Gamma$ using the Kubo formula within a simple tight-binding model. The aim of this calculation was threefold: first, to demonstrate that the resistivity of a disordered metal layer depends on its thickness $L$, when this layer is placed between leads of a higher potential; second, to show that the redistribution of the current-carrying electrons in the $\mathbf{k}_{\|}$space changes with the thickness of the layer and with the distance from the interface due to scattering by disorder; and, third, to demonstrate that the 2CSR model fails to describe the results of accurate calculations for CPP GMR. In the calculation we use a real-space approach proposed in Ref. 16 and generalized to the three-dimensional structures in Ref. 17. Within this approach the conductance is calculated by considering a disordered conductor, connected to two perfect semi-infinite metallic leads. First, one finds the matrix elements of the surface Green's function for the leads. Then, the sample is grown by adding disordered layers onto the left lead and/or onto the right lead. The Green's function of the added layers is recalculated at each step recursively by solving numerically the respective Dyson equation. Once the sample has been fully grown, the last two layers are bonded to each other in order to obtain the Green's function of the full system, which enters the expression for the conductance. By changing the position $l$ of the last layers we investigate the $\mathbf{k}_{\|}$distribution of the conductance at various distances from the leads. With this aim, we transform the basis functions from atomic orbitals $|i\rangle$ to the Bloch functions $\left|\mathbf{k}_{\|}\right\rangle$, thereby obtaining the contribution to the total conductance $\Gamma$ from various transverse momenta, i.e., $\Gamma\left(\mathbf{k}_{\|}, l\right)$. We note that due to current conservation $\Gamma=\Sigma_{\mathbf{k}_{\|}} \Gamma\left(\mathbf{k}_{\|}, l\right)$ is independent of $l$.

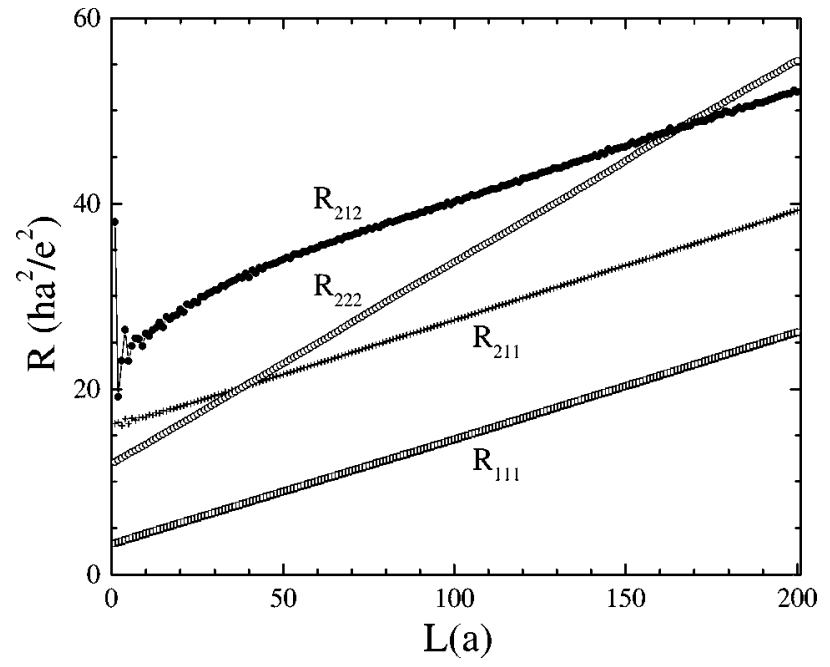

FIG. 1. Resistance of a disordered metal layer versus its thickness $L$ for various metals and leads: opened squares, $R_{111}$, metal 1 $\left(E_{1}=3\right)$, leads of metal 1 ; opened circles, $R_{222}$, metal $2\left(E_{2}\right.$ $=5$ ), leads of metal 2; crosses, $R_{211}$, metal 1, left lead of metal 2, right lead of metal 1; full circles, $R_{212}$, metal 1 , leads of metal 2.

In the calculation we assume that the metals have a simple cubic geometry of lattice parameter $a$ and (001) orientation of atomic layers. The hopping integrals are nonzero only between nearest neighbors with their magnitude set equal to 1. All energies are measured in units of the hopping integral relative to the Fermi energy, which lies at zero. The bulk disorder is introduced according to the Anderson model ${ }^{18}$ as a random variation of the on-site atomic energies with a uniform distribution of standard deviation $\gamma=0.5$. The calculated conductance is averaged over 80 random configurations. The size of the system is extended to infinity in the direction perpendicular to the current by introducing a unit cell of $16 \times 16$ atoms in the transverse direction and imposing periodic boundary conditions.

In order to form a potential-well structure we consider two types of metal, which differ by their on-site atomic energies $E_{m}$, i.e., metal 1 with $E_{1}=3$ and metal 2 with $E_{2}$ $=5$. We denote the resistance and the conductance of disordered metal $j$ placed between leads of metals $i$ and $k$ by $R_{i j k}$ and $\Gamma_{i j k}$, respectively, where $i, j$, and $k$ are 1 or 2 . In the absence of the potential well the resistance of the metal layers $R_{111}$ and $R_{222}$ is a linear function of their thicknesses, which is seen from the open squares and the open circles in Fig. 1 and is the evidence of the Ohmic regime of conductance. The resistivity is a factor of 2 higher for metal 2 than for metal 1, both being in good agreement with those obtained for the same parameters using our previously published weak-scattering model. ${ }^{19}$ The resistances at zero thickness correspond to the ballistic limit and are consistent with the results obtained in Ref. 15.

The linear behavior of the resistance versus the layer thickness changes dramatically in the presence of the potential well. This can be seen from the full circles in Fig. 1, which show the resistance of the metal-1 film placed between the leads of metal 2, i.e., $R_{212}$, so that the potential step $U=E_{2}-E_{1}=2$ is introduced at both interfaces of the film. Noticeable oscillations in the resistance at very small thicknesses are reminiscent of the oscillations in the ballistic 

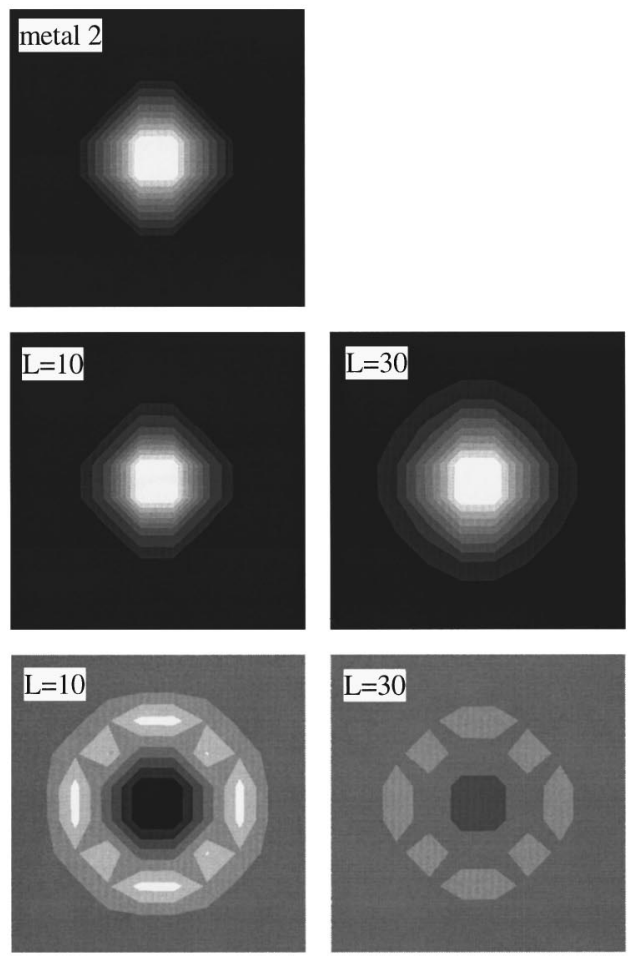
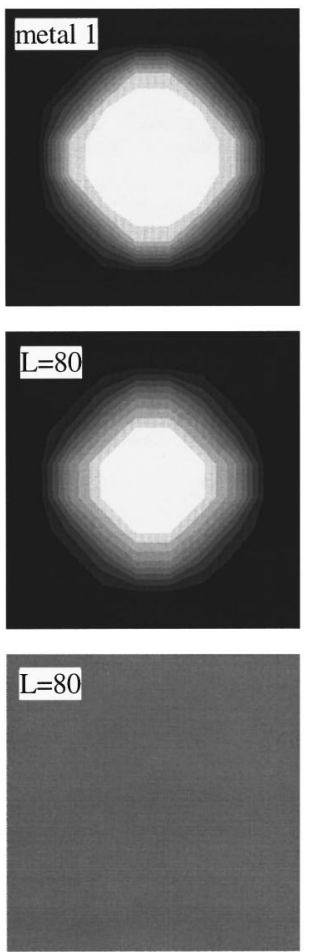

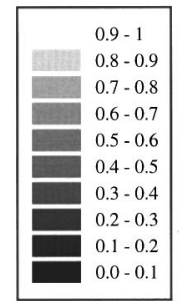

$0.9-1$
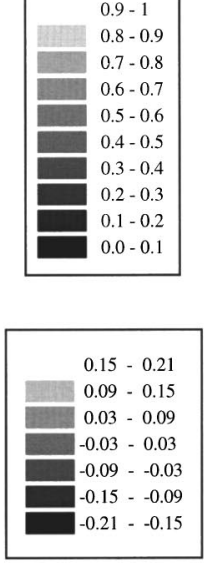

FIG. 2. Normalized conductance distribution within the first Brillouin zone, $-\pi \leqslant k_{x} \leqslant \pi,-\pi$ $\leqslant k_{y} \leqslant \pi$, in the bulk metals 2 and 1 (top panels) and at the middle of the disordered metal-1 layer of various thickness $L$, placed between leads of metal 2 (middlerow panels). The $\mathbf{k}_{\|}$contribution to the difference in the resistance of the first and the second interface (bottom panels). regime of conduction. ${ }^{14}$ The important feature, which is evident from Fig. 1, is that the average slope in the resistance curve, which determines the resistivity, changes with the metal-1 layer thickness. At small thicknesses it is similar to the slope in the resistance curve of metal 2 (compare the solid circles and the open circles in Fig. 1 for $L<50 a$ ). Increasing $L$ results in a decrease in the resistivity (1), which approaches the value of that in metal 1 (compare the solid circles and the open squares in Fig. 1 for $L>100 a$ ). The characteristic length scale for the change in the resistivity is the mean free path. The latter can be estimated from the relation $l_{\mathrm{mfp}} \approx \hbar \mathrm{v} /\left(2 \pi \gamma^{2} n_{F}\right)$, where $\mathrm{v}$ is an average velocity and $n_{F}$ is the density of states per atom at the Fermi energy. Estimating $\mathrm{v}$ from the band dispersions at $k_{x}=k_{y}=k_{z}$, we obtain that $l_{\mathrm{mfp}} \approx 25 a$ in the metal 1 . As seen from Fig. 1, the change in the slope and consequently in the resistivity extends up to a few $l_{\text {mfp }}$ into the bulk metal 1 .

As follows from the crosses in Fig. 1, if the potential step is introduced only at one interface no resistivity change is observed, because no bound states are formed in the disordered layer. The upward shift of the $R_{211}$ curve with respect to the $R_{111}$ curve is the result of the interface resistance. As is clear from the difference $R_{212}-R_{211}$ in Fig. 1, the presence of the bound states causes the conduction to be greatly reduced at small thicknesses of the inserted layer. At large thicknesses the resistances of the first and second interface become equal, as expected (compare $R_{212}-R_{211}$ and $R_{211}$ $-R_{111}$ in Fig. 1 for $L>100 a$ ).

In order to demonstrate that the change in the resistivity is associated with the opening of the new conducting channels, we have calculated the distribution of the conductance between various $\mathbf{k}_{\|}$, as has been described above. The top panels in Fig. 2 show the respective distribution in the first Brillouin zone for the bulk metals 2 and 1. As is obvious from the figure, the number of the conducting channels is much larger for metal 1 than for metal 2, which is reflected by the larger bright area around $k_{\|}=0$ in the right top panel of Fig. 2. The middle-row panels in Fig. 2 show the conductance distribution at the middle of the metal-1 layer for various thicknesses when this layer is placed between the leads of metal 2. The distribution displays a remarkable transformation from that which is similar to the distribution in bulk metal 2 (compare the left-top and left-middle-row panels in Fig. 2) to that which is similar to the distribution in bulk metal 1 (compare the right-top and right-middle-row panels in Fig. 2). Those $\mathbf{k}_{\|}$that correspond to the bound states in the absence of disorder, i.e., the states that lie beyond the bright area in the left-top panel, become contributing to the conduction. The number of conducting channels increases, therefore, with the thickness of the disordered metal, reducing the resistivity of this layer. This is different to what we find for the case when the potential step is set at only one interface. Although the $\mathbf{k}_{\|}$distribution change is similar to that in the potential-well structure (not shown), it is not accompanied by the opening of new conducting channels, because no bound states are present in metal 1 in the latter case. This is evident from the bottom panels in Fig. 2, where we have plotted the $\mathbf{k}_{\|}$contribution to the difference in the resistance of the first and the second interface

$$
\begin{aligned}
\Delta R_{i}\left(\mathbf{k}_{\|}\right)= & {\left[\Gamma_{111}\left(\mathbf{k}_{\|}\right)-\Gamma_{211}\left(\mathbf{k}_{\|}\right)\right] /\left(\Gamma_{111} \Gamma_{211}\right)-\left[\Gamma_{211}\left(\mathbf{k}_{\|}\right)\right.} \\
& \left.-\Gamma_{212}\left(\mathbf{k}_{\|}\right)\right] /\left(\Gamma_{211} \Gamma_{212}\right)
\end{aligned}
$$

The first term in this expression is the resistance of the singe interface at given $\mathbf{k}_{\|}$and the second term is the resistance of the second interface in the presence of the first one. As is clear from the left-bottom panel, at small thicknesses the resistance difference is positive for those $\mathbf{k}_{\|}$that lie beyond the conduction region for metal 2 (compare with the left-top panel). These $\mathbf{k}_{\|}$contribute to the resistance of the single interface, but they do not contribute to the resistance of the second interface within the potential-well structure. With in- 


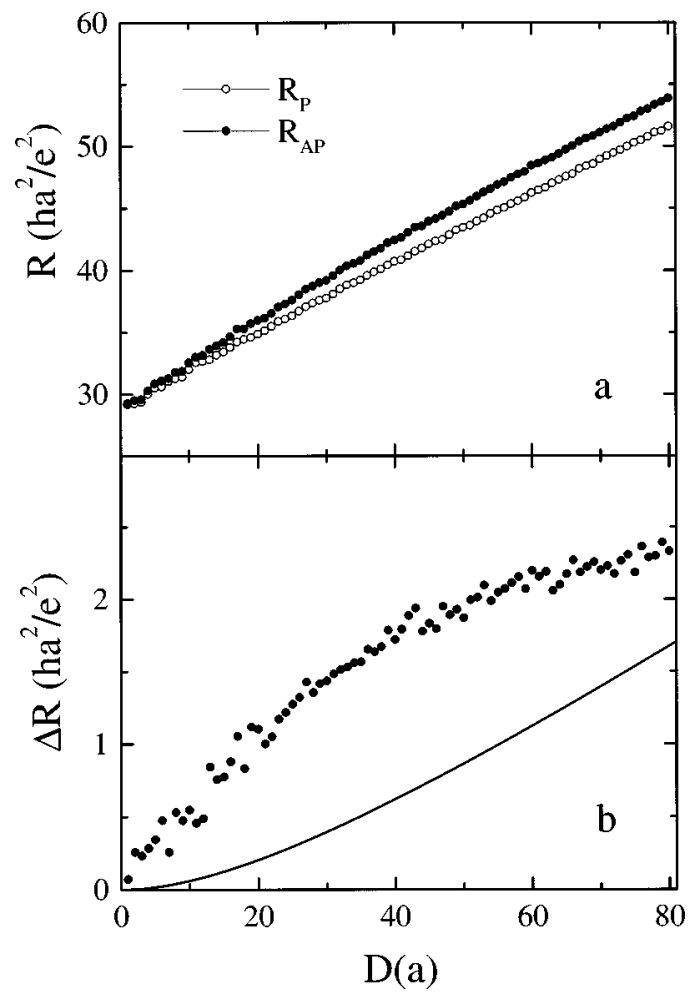

FIG. 3. CPP GMR: (a) resistance of the trilayer as a function of thickness $D$ of the ferromagnetic layers for $P$ and AP magnetizations; (b) difference in the resistance for $P$ and AP magnetizations, obtained from the accurate calculation (circles) and from the twocurrent series-resistor model (solid line).

creasing thickness the contrast of the plot reduces, reflecting the fact that the bound states become involved in the conduction with $\Delta R_{i}\left(\mathbf{k}_{\|}\right)$disappearing eventually at large thicknesses, as expected.

Finally, we calculated the CPP GMR in a disordered magnetic trilayer, which was constructed from two ferromagnetic layers of the same thickness $D$ separated by a nonmagnetic spacer layer of $20 a$. The two spin bands in the ferromagnets were treated independently, the up-spin band having an onsite energy $E_{\uparrow}=3$ and the down-spin having an on-site energy $E_{\downarrow}=5$. The metal 1 was used for the spacer layer and the metal 2 was used for the leads. The conductance $\Gamma$ was calculated for the parallel $(P)$ and antiparallel (AP) magnetizations of the ferromagnetic layers as a function of their thickness $D$ in the spirit of the experiments performed in Ref. 11. As is seen from Fig. 3(a), the resistance of the trilayer is a weak nonlinear function of the ferromagnetic layer thickness, the nonlinearity being more pronounced for the AP configuration. This behavior is a consequence of the quantum-well states, which enhance the resistivity defined by Eq. (1) at small thicknesses, the enhancement being stronger for the AP configuration due to the narrower potential well compared to the $P$ configuration.

As is obvious from Fig. 3(b), the difference in the resistance $\Delta R$ for the AP and $P$ configurations (circles), behaves differently from that predicted within the 2CSR model (solid line), according to which

$$
\Delta R(L)=\frac{1}{2}\left(\rho_{\uparrow}-\rho_{\downarrow}\right)^{2} D^{2} /\left(r+\rho_{\uparrow} D+\rho_{\downarrow} D\right) .
$$

Here $\rho_{\uparrow}$ and $\rho_{\downarrow}$ are the resistivities of the up-spin and downspin electrons, respectively, and $r$ is the summed resistance of the leads, the interfaces, and the spacer layer. The $\rho_{\uparrow}$ and $\rho_{\downarrow}$ were obtained from the resistance-thickness curves for metals 1 and 2, shown in Fig. 1, and $r$ was found by extrapolating the curves in Fig. 3(a) to zero thickness. The deviation between the 2CSR predictions and our more accurate calculation is sizeable even at thicknesses greater than the mean free path, because of the contribution to conductance from the electronic states with different velocities and consequently different decay lengths. We conclude, therefore, that, in general, the 2CSR model is a poor description of CPP GMR. This fact is illustrated by recent experiments, ${ }^{20}$ which show that the magnitude of the CPP GMR depends strongly on the order of alternating thick and thin magnetic layers in a multilayer, which is not predicted within the 2CSR model. We believe that the mechanism proposed in the present paper is the key to understanding these experiments and are currently extending our theory to include a realistic band structure of the multilayer.

In conclusion, we have shown that the resistivity of a metal film is enhanced compared to the bulk resistivity, if this film is placed in a potential well and the electric current flows perpendicular to the planes. The effect results from the formation of quantum-well bound states, which reduce the number of conducting channels in the film. Scattering by disorder redistributes current-carrying electrons between various transverse momenta, which opens new conducting channels and changes the quantum-well resistivity at thicknesses comparable with the mean free path. This makes the mean free path an important parameter, which has to be included in any accurate model for the CPP GMR, rather than ignored as within the two-current series-resistor model.

This research was supported by Hewlett-Packard Laboratories, Palo Alto. The authors are thankful to Mark Howson, Bryan Hickey, and Chris Marrows for the discussions, which stimulated the present study. E. Yu. T. is grateful to Peter Levy for his very useful comments and Tchavdar Todorov for the helpful discussion. The computations were performed in the Materials Modelling Laboratory at the Department of Materials, University of Oxford, on an HP Exemplar V-class computer jointly funded by HP and HEFCE through the JREI scheme.
${ }^{1}$ M. N. Baibich, J. M. Broto, A. Fert, F. Nguyen Van Dau, F. Petroff, P. Eitenne, G. Greuzet, A. Friederich, and J. Chazelas, Phys. Rev. Lett. 61, 2472 (1988).

${ }^{2}$ W. P. Pratt, Jr., S.-F. Lee, J. M. Slaughter, R. Loloee, P. A. Schroeder, and J. Baas, Phys. Rev. Lett. 66, 3060 (1991).

${ }^{3}$ S. Zhang and P. M. Levy, J. Appl. Phys. 69, 4786 (1991).
${ }^{4}$ H. E. Camblong, S. Zhang, and P. M. Levy, Phys. Rev. B 47, 4735 (1993)

${ }^{5}$ T. Valet and A. Fert, Phys. Rev. B 48, 7099 (1993).

${ }^{6}$ R. Q. Hood and L. M. Falicov, Phys. Rev. B 46, 8287 (1992).

${ }^{7}$ J. Barnas and A. Fert, Phys. Rev. B 49, 12835 (1994).

${ }^{8}$ S. Zhang and P. M. Levy, Phys. Rev. B 57, 5336 (1998). 
${ }^{9}$ A. Vedyayev, N. Ryzhanova, B. Dieny, P. Dauguet, P. Gandit, and J. Chaussy, Phys. Rev. B 55, 3728 (1997).

${ }^{10}$ Q. Yang, P. Holody, S.-F. Lee, L. L. Henry, R. Loloee, P. A. Schroeder, W. P. Pratt, Jr., and J. Bass, Phys. Rev. Lett. 72, 3274 (1994).

${ }^{11}$ S. D. Steenwyk, S. Y. Hsu, R. Loloee, J. Bass, and W. P. Pratt, Jr., J. Magn. Magn. Mater. 170, L1 (1997).

${ }^{12}$ S. Dubois, L. Piraux, J. M. George, K. Ounadjela, J. L. Duvail, and A. Fert, Phys. Rev. B 60, 477 (1999).

${ }^{13}$ D. M. Edwards, J. Mathon, R. B. Muniz, and M. S. Phan, Phys. Rev. Lett. 67, 493 (1991).
${ }^{14}$ J. Mathon, M. Villeret, and H. Itoh, Phys. Rev. B 52, 6983 (1995).

${ }^{15}$ K. M. Schep, P. J. Kelly, and G. E. W. Bauer, Phys. Rev. B 57, 8907 (1998).

${ }^{16}$ P. A. Lee and D. S. Fisher, Phys. Rev. Lett. 47, 882 (1981).

${ }^{17}$ T. N. Todorov, Phys. Rev. B 54, 5801 (1996).

${ }^{18}$ P. W. Anderson, Phys. Rev. B 23, 4828 (1981).

${ }^{19}$ E. Yu. Tsymbal and D. G. Pettifor, Phys. Rev. B 54, 15314 (1996).

${ }^{20}$ D. Bozec, M. A. Howson, B. J. Hickey, S. Shatz, and N. Wiser, cond-mat/9906410 (unpublished). 